\title{
Association of serum hormone levels with acne vulgaris: Low estradiol level can be a pathogenetic factor in female acne
}

\author{
Neslihan Akdoğan', Sibel Doğan², Nilgün Atakan², Başak Yalçın¹
}

${ }^{1}$ Department of Dermatology and Venereology, Ankara Numune Training and Research Hospital, Altindağ, Ankara, Turkey, ${ }^{2}$ Department of Dermatology and Venereology, Hacettepe University, Faculty of Medicine, Sihhiye, Ankara, Turkey

Corresponding author: Dr. Neslihan Akdoğan, E-mail: nslakdogan@gmail.com

\begin{abstract}
Background: Acne vulgaris is a chronic disease with a multifactorial pathogenesis. Speculative data has been shown concerning acne vulgaris and hormones, obesity, inflammatory, metabolic disorders. Aims: Our study aimed to evaluate hormone levels, inflammatory, metabolic parameters of patients with acne vulgaris. Materials and Methods: 136(male/ female:42/94) participants including 68 patients and 68 age and sex-matched controls were enrolled retrospectively. Age, gender, height, weight, body mass index (BMI), systolic (SBP) and diastolic (DBP) blood pressure, serum lipid profile (SLP), fasting blood sugar (FBS), C-reactive protein (CRP) and red cell distribution width (RDW) parameters were evaluated for patients and controls. Patients were questioned about the type and duration of acne, previous treatments, presence of acne vulgaris and other types of acne in first-degree relatives. Females (47patients, 47 controls) were evaluated for follicle-stimulating hormone (FSH), luteinizing hormone (LH), progesterone (PRO), estradiol (EST), prolactin (PRL) and total testosterone (TT) levels collected on the early follicular phase of menstruative cycle. Results: There was no statistically significant difference between patients and controls regarding BMI, SBP, DBP, SLP, FBS, CRP, RDW parameters (all p values $>0.05$ ). Moreover, serum FSH, LH, PRO, PRL, TT levels showed no statistically significant difference between female patients and female controls (all p values $>0.05$ ). However, female patients had statistically significant lower levels of serum EST than female controls. Conclusion: Low serum EST levels can be observed in female acne patients. Further investigation is required to define the association between serum EST and acne. This may also bring a new approach to the treatment of female acne vulgaris by estrogen containing hormone therapies.
\end{abstract}

Key words: Acne vulgaris; Estradiol; Female; Hormone

\section{INTRODUCTION}

Acne vulgaris is one of the most common skin diseases affecting the pilosebaceous unit [1]. Multifactorial pathogenesis of acne vulgaris includes inflammation [2,3], dietary intake [4-6], hormones [7-9], especially androgens [2,3], metabolic imbalances, insulin resistance and obesity $[10,11,12]$. Genetic background [13] is also accepted to play a role in the pathogenesis of acne. Despite several studies on acne pathogenesis, possible relationships between predisposing and aggrevating factors are poorly understood. Recently, acne is suggested as a visible indicator of systemically exaggerated mammalian target of rapamycin complex 1 (mTORCl) signaling. mTORCl is a protein complex which plays a crucial role in protein and lipid synthesis, cell growth and proliferation [14]. In acne vulgaris, metabolic deviation due to increased mTORCl signal pathway occurs resulting in mTORCl-driven diseases, such as obesity, arterial hypertension, insulin resistance, type 2 diabetes mellitus, and even cancer and Alzheimer's disease $[12,15]$.

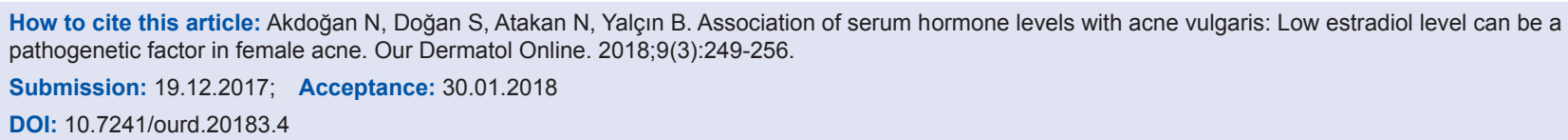


A probable association between acne and inflammation has long been postulated. Propionibacterium acnes (P. acnes), which is a bacteria involved in the pathogenesis of acne, can induce IL-1, IL-8, TNF- $\alpha$ leading to subsequent secretion of high amounts of CRP from the liver [16,17]. Thus, locally increased levels of inflammatory cytokines may lead to elevation of serum CRP levels. RDW is also an inflammatory biomarker which shows correlation with CRP. It is an independent risk factor in patients with coronary artery disease and heart failure probably reflecting an underlying chronic inflammation [18]; however, the link between acne and RDW remains uncertain.

Studies are able to show certain effects of BMI on acne vulgaris development and dietary interventions are proposed to play a role in the management of acne [19]. High-glycemic index diets play a role in the induction and exacerbation of acne contributing to overstimulation of sebum production, P. acnes overgrowth, biofilm formation while low-glycemic index diets reduce the severity of acne [5]. Furthermore, one of the diseases that particularly shows strong relation between BMI and acne is polycystic ovary syndrome (PCOS). Weight loss in PCOS patients is suggested as an effective method of restoring ovulation and menstruation by reducing androgen levels and thereby improving ovarian function. These effects are related to reduction of insulin levels and improvement of insulin resistance and thereby decreasing acne severity [20,21] .

Human sebum is composed of triglycerides, diglycerides, free fatty acids, wax esters, squalene with some cholesterol and cholesterol esters [22]. Changes in the lipid content of sebum and increased sebum production have a role in the development of acne [23]. Whether serum lipids are elevated in patients with acne or not is still a matter of debate. The role of androgens in acne pathogenesis is evident, increase in androgen levels during puberty stimulates sebum production, increases size of the sebaceous glands, stimulates keratinocyte proliferation in sebaceous gland duct and acro-infundibulum[2]. Testosterone, an androgenic hormone, is converted to its more potent form called dihydrotestosterone (DHT) through the action of the enzyme $5 \alpha$-reductase (5-ARD) causing an excess production of sebum [20]. Although the pathogenesis of acne is closely related with androgens, androgen levels were within normal ranges in many patients [24]. There is limited data about androgenic (testosterone) and non-androgenic hormones (FSH, LH, PRO, EST, PRL) on acne. FSH and LH which are secreted by the anterior pituitary gland stimulate gonads for androgen secretion. Especially, PCOS patients who have increased LH/FSH ratio have acne as a common clinical comorbidity [20]. Estrogens have several effects on sebum such as decrease in sebaceous gland sizes and sebum production in high doses $[25,26]$. Progesterone inhibits the activity of 5-ARD and prevents conversion of testosterone into DHT that probably help reducing sebaceous gland activity. Prolactin which is a hormone mainly involved in the milk production also increases the activity of 5-ARD.

The aim of the present study was to evaluate hormone levels, inflammatory and metabolic parameters of acne vulgaris patients. For this aim, age and gender characteristics, anthropometric measurements (height, weight and BMI), SBP and DBP, laboratory parameters of SLP, FBS, CRP, RDW were evaluated in all participants. Acne vulgaris type, duration of acne, previous acne treatments, presence of acne vulgaris and other types of acne in first-degree relatives were recorded in patients. Additionally, serum levels of FSH, LH, PRO, EST, PRL and TT were assessed in female patients and female controls.

\section{MATERIALS AND METHODS}

\section{Study Protocol}

A total of 136 participants; 68 acne vulgaris patients (M/F: 21/47) and 68 age and sex-matched controls (M/F: 21/47) who were older than 18 years of age were retrospectively enrolled for the study. Age and gender characteristics, anthropometric measurements including height $(\mathrm{kg})$, weight $(\mathrm{m}), \mathrm{BMI}\left(\mathrm{kg} / \mathrm{m}^{2}\right)$, systolic (normal 90-120 mmHg) and diastolic (normal 60$80 \mathrm{mmHg}$ ) blood pressure, laboratory analyses of SLP (total cholesterol, (TC, normal 0-200 mg/dl), highdensity lipoprotein cholesterol (HDL-C, normal 40$60 \mathrm{mg} / \mathrm{dl}$ ), low-density lipoprotein cholesterol (LDL-C, normal 0-99 $\mathrm{mg} / \mathrm{dl}$ ) and triglycerides (TG, normal 30$150 \mathrm{mg} / \mathrm{dl}$ )), FBS (normal 70-110 mg/dl), CRP (normal 0-5 mg/dl) and RDW (normal 11.6-14.6\%) parameters were evaluated from all participants. Acne vulgaris type, duration of acne, previous acne treatments, presence of acne vulgaris and other types of acne in first-degree relatives were evaluated for each patient. Body mass index was calculated by using Quetelet index as dividing weight by the square of height [27]. Four categories are created based on the calculated BMI scores: 1. Underweight: Lower than 18.50; 2. Normal 
range: 18.50-22.99; 3. Overweight: 23.00-24.99; 4. Obese: 25 or higher [28]. The recorded blood pressure values were measurements taken by a blood pressure monitor after at least resting 10 minutes (ERKA. The Original, VARIO, blood pressure monitor, Germany). Five $\mathrm{ml}$ of peripheral venous blood was collected from all participants by using biochemistry tubes (BD Vacutainer ${ }^{\circledR}$ SST II Plus plastic serum tube, 367955 - 13 $\times 100 \mathrm{~mm} \times 5.0 \mathrm{~mL}$, BD Diagnostics - Preanalytical Systems, USA) after 8 hours of fasting for evaluation of SLP, FBS, CRP (AU5800 Clinical Chemistry System; Beckman Coulter Inc., USA) and by using ethylenediaminetetraacetic acid (EDTA) tubes (EDTA. K3 GD020EK3 blood collection tube, $13 \times 75 \mathrm{~mm} \times$ $2.0 \mathrm{~mL}$ ) for evaluation of RDW (Sysmex ${ }^{\circledR}$ XS-1000i Automated Hematology Analyzer, Sysmex America, Inc., USA). SLP, FBS and CRP levels were measured by photometric, hexokinase and turbidimetry methods, respectively.

Furthermore, female participants $(\mathrm{n}=94)$ including patients $(n=47)$ and controls $(n=47)$ were evaluated for serum FSH (normal 2.5-10.2 mIU/mL), LH (normal 1.9-12.5 mIU/mL), EST (normal 11-63 pg/mL), PRO (normal 0.15-1.40 ng/mL), PRL (normal 2.8-29.2\%) and TT (normal 21-118 ng/mL) levels which were performed on the early follicular phase (2-5. days) of menstruative cycle. On the early follicular phase of menstruative cycle, $5 \mathrm{ml}$ of peripheral venous blood was collected from female patients and female controls to analyze serum FSH, LH, PRO, PRL, EST and TT levels by using chemiluminescence enzyme immunoassay method (UniCel ${ }^{\circledR}$ DxI 800 Immunoassay System; Beckman Coulter Inc., Clinical Diagnostics Division, Brea, CA, USA).

Type of acne vulgaris was defined as comedonal, mild papulopustular, moderate papulopustular and nodulocystic [1]. The duration of acne disease was expressed as months. Patients were grouped under 5 categories for previous acne treatments (Group 1: No previous treatment, Group 2: Having previously received topical therapy alone. Group 3: Having previously received topical therapy and antibiotherapy. Group 4: Having previously received oral isotretinoin. Group 5: Having previously received both topical therapy, antibiotherapy and oral isotretinoin as combination or at different times). Menstruative cycle pattern of the female participants is examined in 7 groups: 1. Premenarche: No menarche before 16 years of age, 2. Primary amenorrhea: Menarche was not started after 16 years of age, 3 . Secondary amenorrhea:
The absence of menstruation for 180 days or more, 4. Oligomenorrhea: An average menstrual cycle of 42 to 180 days, 5. Polymenorrhea: An average menstrual cycle of 21 days or less, 6. Regular menstrual cycles: An average menstrual cycle of 22 to 41 days, 7 . Irregular menstrual cycles with an average menstrual cycles of 22 to 41 days and two or more menstrual cycles with a length of less than 22 or more than 41 days during the past year [29].

Exclusion criteria for this study were pregnancy, lactation, cigarette smoking, presence of diabetes mellitus, hypertension, hyperlipidemia, atherosclerotic heart disease, systemic diseases affecting CRP levels (rheumatic disease, inflammatory bowel disease, cancer, chronic infections), anemia (defined as hemoglobin levels $<12.0 \mathrm{~g} / \mathrm{dL}$ in women and $<13.0 \mathrm{~g} /$ $\mathrm{dL}$ in men according to the World Health Organization (WHO) [30]), systemic anti-inflammatory treatment for any reason in the past 1 month, presence of primary gonadal failure. Participants using medications known to affect hormone levels (ovulation induction therapies, high dose testosterone, PRO and estrogen treatments), lipid metabolism (statins, thiazide diuretics, estrogen and androgen preparations, selective estrogen receptor modulators, antidiabetics, orlistat, corticosteroids, cyclosporine, mitotane, isotretinoin, antipsychotics, antiretrovirals), sugar metabolism (oral antidiabetics, insulin, corticosteroids, barbiturates, thiazide diuretics, OCS, decongestants, niacin, olanzapine) and serum CRP levels (cyclooxygenase inhibitors, platelet aggregation inhibitors, lipid reducing drugs, beta adrenoceptor antagonists, angiotensin-convertingenzyme inhibitors, angiotensin receptor blockers, vitamine E) were also excluded from this study. None of the patients received treatment for acne during the laboratory examination. Control group subjects were chosen from individuals without medical or family history of acne. All parameters used in the study were evaluated from the database of the hospital.

\section{Data Analysis}

Statistical Package for the Social Sciences (SPSS Inc., Chicago, IL, USA) version 20.0 was used for all statistical analyses. Results were expressed as mean \pm standard deviation (SD) and median \pm interquartile range (IR). Shapiro-Wilk test was used to understand whether numeric variables distribute normally or not. When parametric assumptions were provided, independent sample t-test was used. Differences in numeric variables between independent groups 
were analyzed by using Mann-Whitney $U$ test when parametric assumptions were not provided. The ci-square test was used to determine whether there were differences in categorical variables between independent groups or not. Relationships between the quantitative variables were calculated by using the Pearson's and Spearman's correlation coefficient. Numeric variables which are not normally distributed were analyzed by Kruskal Wallis test while normally distributed numeric variables are examined by oneway analysis of variance (ANOVA) test. $\mathrm{p}<0.05$ was considered to be statistically significant.

\section{Ethics Statement}

The study protocol was approved by the Institutional Ethics Review Board at the Ankara Numune Training and Research Hospital with number of E-16-999 and was conducted according to the ethical principles of the Declaration of Helsinki.

\section{RESULTS}

Sixty-eight patients (M/F: 21/47) with acne vulgaris and 68 healthy controls (M/F: 21/47) were included in this study. Age, gender, height, weight, BMI, SBP, DBP, SLP, FBS, CRP and RDW parameters were evaluated for each individual. Acne vulgaris type, duration of acne, previous acne treatments, presence of acne vulgaris and other types of acne in first-degree relatives were recorded in patients. Female participants $(n=94,47$ female patients and 47 female controls) were evaluated for their hormone profile consisting of serum FSH, LH, PRO, EST, PRL and TT levels which were collected on the early follicular phase of menstruative cycle. Comparisons of the parameters analyzed in this study are summarized in Table 1 and Table 2. Patient and control groups did not have any statistically significant difference according to age $(p>0.05) . \mathrm{N}=10(14.7 \%)$ of the patients had comedonal, $\mathrm{N}=10(14.7 \%)$ patients had mild papulopustular, $\mathrm{N}=34(50 \%)$ had moderate papulopustular and $\mathrm{N}=14(20.6 \%)$ had nodulocystic acne. $\mathrm{N}=35(51.5 \%)$ patients had never been treated before for acne while $\mathrm{N}=10(14.7 \%)$ of the cases used topical therapy alone, $\mathrm{N}=15(22.1 \%)$ patients used topical therapy and antibiotherapy, $\mathrm{N}=7$ (10.3\%) patients used oral isotretinoin and one patient had both previous treatments of topical therapy, antibiotherapy and oral isotretinoin. The median duration of the acne disease was $24 \pm 46.5$ months (median \pm IR, range: 0-264 months). Thirty of the 68 patients had history of acne in first-degree relatives. Seventy-eight (82.9\%) of the 94 females had regular menstrual cycles taking place in group 6, 7 (7.5\%) was in group 4, 3 (3.2\%) in group 5 , $6(6.4 \%)$ in group 7 , and no female had premenarche or secondary amenorrhea. Forty patients $(58.8 \%)$ and 32 controls $(47.1 \%)$ had BMI scores within normal ranges whereas 10 patients $(14.7 \%)$ and 15 controls $(22.1 \%)$ had scores greater than 25 indicating category 4 as obese. No statistically significant difference was detected between mean BMI scores of the patients and controls which were $22.4 \pm 2.96$ and $21.9 \pm 4.65 \mathrm{~kg} /$ $\mathrm{m}^{2}$, respectively $(p>0.05)$. Comparison of patient and control groups revealed that there was no statistically significant difference between SBP, DBP values and TC, TG, HDL, LDL, FBS, CRP, RDW levels (all $p$ values $>0.05$, Table 1). Moreover, serum FSH, LH, PRO, PRL and TT levels showed no statistically significant difference between female patients and female controls (all $p$ values $>0.05$, Table 2 ). However, female acne vulgaris patients had statistically significant lower levels of serum EST (median \pm IR, $33.6 \pm 16.62 \mathrm{ng} / \mathrm{mL}$ ) than female controls (median $\pm \mathrm{IR}, 43.3 \pm 17.23 \mathrm{ng} / \mathrm{mL}$ ) $(p=0.015$, Table 2$)$.

\section{DISCUSSION}

In this study, we showed that low serum EST levels can be expected in female patients with acne. BMI, SBP, DBP parameters and SLP, FBS, CRP, RDW, FSH, LH, PRO, PRL and TT levels did not show any association with acne vulgaris.

The effects of estrogens on acne pathogenesis is not very clear. Estradiol, the primary female sex hormone, is the evaluated form in this study and known as the major active estrogen form during human female reproductive years in terms of absolute serum levels and estrogenic activity. In general, while androgens contribute to the development of acne through increase in sebum production, estrogens, in sufficient amounts, have inhibitory effects on acne through suppression of sebum production [31]. The effect of estrojen on acne are through three different mechanisms, including opposition of androgens within the sebaceous glands, inhibition of gonadal androgen production via negative feedback mechanism on gonadotrophin release and effects on genes which play a role in sebaceous gland growth and lipid production [32]. But, estrogen effect on sebum production and acne development is not yet well understood since some women experience a deterioriation of acne during high estrogen periods like 
Table 1. Clinical and laboratory findings of 68 acne vulgaris patients and 68 healthy controls.

\begin{tabular}{|c|c|c|c|c|}
\hline Parameters & $\begin{array}{l}\text { Patients }(n=68)(\text { mean } \pm S D \\
\text { or median } \pm I R)\end{array}$ & $\begin{array}{c}\text { Controls }(\mathrm{n}=68) \\
(\text { mean } \pm S D \text { or } \\
\text { median } \pm I R)\end{array}$ & $p$ value & $\begin{array}{l}\text { Normal } \\
\text { range }\end{array}$ \\
\hline Gender (M/F) & $21 / 47$ & $21 / 47$ & & \\
\hline Age (years) & $20.5 \pm 5$ & $21.0 \pm 5$ & 0.901 & \\
\hline Height $(m)$ & $1.65 \pm 7.7$ & $1.67 \pm 8.3$ & 0.249 & \\
\hline Weight (kg) & $60.9 \pm 9.5$ & $61.9 \pm 10.9$ & 0.432 & \\
\hline $\mathrm{BMI}^{*}\left(\mathrm{~kg} / \mathrm{m}_{2}\right)$ & $22.4 \pm 2.96$ & $21.9 \pm 4.65$ & 0.902 & $18.50-22.99$ \\
\hline $\mathrm{SBP}(\mathrm{mmHg})$ & $110 \pm 20$ & $110 \pm 20$ & 0.473 & $90-120$ \\
\hline DBP (mmHg) & $70 \pm 20$ & $70 \pm 20$ & 0.546 & $60-80$ \\
\hline FBS (mg/dl) & $97 \pm 10.8$ & $97 \pm 12.8$ & 0.976 & $70-110$ \\
\hline CRP (mg/dl) & $1.97 \pm 1.58$ & $1.90 \pm 1.4$ & 0.102 & $0-5$ \\
\hline TC (mg/dl) & $160.0 \pm 44.3$ & $158.5 \pm 49.3$ & 0.747 & $0-200$ \\
\hline $\mathrm{TG}(\mathrm{mg} / \mathrm{dl})$ & $73 \pm 35.3$ & $78 \pm 64$ & 0.094 & $30-150$ \\
\hline HDL-C (mg/dl) & $52.0 \pm 21$ & $53.5 \pm 17.8$ & 0.403 & $40-60$ \\
\hline LDL-C (mg/dl) & $78.0 \pm 25.3$ & $83.5 \pm 33.3$ & 0.233 & $0-99$ \\
\hline RDW-CV (\%) & $13.2 \pm 1.07$ & $13.1 \pm 0.97$ & 0.065 & $11.6-14.6$ \\
\hline
\end{tabular}

${ }^{*}$ Data is reported as mean \pm SD for BMI due to its normal distribution, Independent Samples $t$ test. Apart from BMI, data are reported as median $\pm \mathrm{IR}$,

Mann-Whitney $\mathrm{U}$ test. $\mathrm{SD}=$ standard deviation; IR=Interquartile range; $\mathrm{M}=$ male; $\mathrm{F}=$ female; $\mathrm{BMI}=$ body mass index; $\mathrm{SBP}=$ systolic blood pressure; $\mathrm{DBP}=$ diastolic blood pressure; $\mathrm{FBS}=$ fasting blood sugar; $\mathrm{CRP}=\mathrm{C}$-reactive protein; $\mathrm{TC}=$ total cholesterol; $\mathrm{TG}=$ triglycerides; $\mathrm{HDL}-\mathrm{C}=$ high-density lipoprotein cholesterol; LDL-C=low-density lipoprotein cholesterol; RDW-CV=red cell distribution width.

Table 2. Laboratory findings of 47 female acne vulgaris patients and 47 female healthy controls.

\begin{tabular}{lcccc}
\hline Parameters & $\begin{array}{c}\text { Patients }(\mathbf{n}=\mathbf{4 7}) \\
\text { (mean } \pm \text { SD or } \\
\text { median } \pm \text { IR) }\end{array}$ & $\begin{array}{c}\text { Controls }(\mathbf{n}=\mathbf{4 7}) \\
\text { (mean } \pm \text { SD or } \\
\text { median } \pm \text { IR) }\end{array}$ & $\begin{array}{c}\text { Normal } \\
\text { Range }\end{array}$ \\
\hline $\mathrm{FSH}(\mathrm{mlU} / \mathrm{mL})$ & $6.92 \pm 1.54$ & $6.79 \pm 1.91$ & 0.724 & $2.5-10.2$ \\
$\mathrm{LH}(\mathrm{mlU} / \mathrm{mL})$ & $4.64 \pm 2.75$ & $4.66 \pm 2.01$ & 0.823 & $1.9-12.5$ \\
$\mathrm{EST}(\mathrm{pg} / \mathrm{mL})$ & $33.6 \pm 16.62$ & $43.3 \pm 17.23$ & 0.015 & $11-63$ \\
$\mathrm{PRO}(\mathrm{ng} / \mathrm{mL})$ & $0.54 \pm 0.42$ & $0.53 \pm 0.33$ & 0.832 & $0.15-1.40$ \\
$\mathrm{PRL}(\%)$ & $13.1 \pm 11.1$ & $11.67 \pm 9.28$ & 0.073 & $2.8-29.2$ \\
$\mathrm{TT}(\mathrm{ng} / \mathrm{mL})$ & $40.65 \pm 261.7$ & $53.1 \pm 267.4$ & 0.212 & $21-118$ \\
\hline
\end{tabular}

${ }^{*}$ Data is reported as mean \pm SD for FSH due to its normal distribution, Independent Samples $t$ test. Apart from FSH, data are reported as median \pm IR, Mann-Whitney $\mathrm{U}$ test. $\mathrm{SD}=$ standard deviation; IR=Interquartile range; $\mathrm{FSH}=$ follicle-stimulating hormone; $\mathrm{LH}=$ =luteinizing hormone; $\mathrm{EST}=e s t r a d i o l ;$ $\mathrm{PRO}=$ progesterone; $\mathrm{PRL}=$ prolactin; $\mathrm{TT}=$ total testosteron.

pregnancy. Moreover, in recent years, a new concept has emerged as adult female acne in which the disease is present after the age of 25 years in women and hormonal therapies containing estrogen preparates are highly effective for those via opposition of the effects of androgens on pilosebaceous units and sebaceous glands even in patients without hormonal abnormalities. Combined oral contraceptives, containing estrogen and progestin, are used for this purpose to inhibit ovarian androgen production, decrease activation of androgen receptors on sebaceous glands and increase sex hormone-binding globulin in the liver [32] which leads decrease in circulating levels of free testosterone. In studies regarding these patients, efficacy of hormonal therapy is based on improvement of acne evaluated by investigator and patient, reduction in lesion counts and severity of acne grades [33] and not based on hormone levels. But, it is known that serum estradiol and androgen levels will decrease in women receiving oral contraceptives. However, we don't know whether female acne patients who benefit from hormonal therapies have initially lower serum estrogen levels compared with those of female acne patients with normal serum estrogen levels. For this reason, studies are required to compare initial and final hormone levels in female acne patients who do and do not benefit from hormone therapies. The relationship between serum EST levels and acne was demonstrated by Wei et al. as serum EST levels was significantly different between female cases with acne and female controls except males. This data was also consistent with the report of Arora et al. [9] revealing that high serum EST levels protects females against the onset of adolescent acne [34]. In accordance with previous data, the current study showed significantly low serum levels of EST in female patients when compared to female healthy controls. The role of estrogen rich foods, drugs containing high amounts of estrogen such as pills, skin patches, topical agents in the treatment of acne may be speculative and contradictive. Similarly, the role of estrogen decreasing conditions such as aging, menopause and some drugs in acne may be questionable too. 
Although androgen hormones significantly play a role in the pathogenesis of acne, serum androgen levels were found to be normal in many patients with acne [24]. In some reports, the effects of androgens on acne development is connected to the androgen receptors instead of its levels suggesting an increased receptor sensitivity to androgens in acne prone skin [35]. A study including 26 patients and 21 controls from Turkey stated that severe acne itself is not related with hyperandrogenism suggesting that it may not be necessary to measure serum androgens in women with severe acne in the absence of other manifestations of hyperandrogenism, such as hirsutism and menstrual irregularity [11]. However, the improvement of acne by anti-androgens [1], increased incidence of acne in patients with PCOS[20] and congenital adrenal hyperplasia who have higher levels of androgens indicate the role of androgens in the pathogenesis of acne. Hormonal therapies will also be useful for female acne patients both in those with hyperandrogenism and without hyperandrogenism [31]. The only androgen measured in this work was serum TT about which we were unable to find any significant differences in serum levels between two female groups at all. Serum TT level was detected on the early follicular phase, perhaps this was the reason why we could not have found any relation with its level and acne.

Testosterone is converted to its more potent form DHT by 5-ARD enzyme causing an excess production of sebum whereas PRO inhibits the activity of this enzyme and prevents turning testosterone into DHT. So, PRO itself might be expected to reduce sebaceous gland activity. But, PRO effect on acne still remains unclear. Although some studies show that PRO can reduce androgen effects by inhibiting 5-ARD enzyme or androgen receptors[36], the fluctuation of sebum production in women during menstrual cycle and premenstrual cyclic flare has partly been associated with PRO and some progestins lead an exacerbation of acne by interacting with androgen receptors [37,38]. Although PRO's unclear effect on acne, combined oral contraceptives which are used for female acne treatment include both an estrogen and a progestin product to decrease the risk of endometrial cancer associated with unopposed estrogens. Due to the fact that many progestins aggravate acne, new generation progestins with lower androgenic activity have began to be used for acne treatment [32]. The relationship between serum PRO levels and acne was demonstrated by Bakry et al. that no significant association between serum PRO levels and the severity of acne was found although a statistically significant difference in serum PRO levels between patients and controls was recorded [39]. Arora et al. also found higher serum PRO levels in patients than that in controls correlating with serum cholesterol levels supporting the fact that cholesterol is the precursor of PRO [40]. In our study, there was no statistically significant difference between female patients and controls in terms of serum PRO levels. This discrepancy between our study and other studies could be explained by analysis of hormone levels on different days of the menstruative cycle and the different profile of the target group.

No significant association was detected between serum FBS levels and acne in this study. Emiroğlu et al. showed that fasting blood glucose levels were not statistically different between 246 patients with acne and 156 healthy controls despite the fact that there was a highly significant difference in terms of fasting insulin levels between these groups suggesting insulin resistance in patients [10]. Furthermore, Kaymak et al. also indicated that there was no significant difference of serum glucose level between patients with acne and control subjects [41]. Contrarily, another study from Turkey showed that FBS levels were significantly higher in patients than that in controls [42].

Many studies have pointed out a possible link between BMI and acne [12]. Seleit et al. studied 60 patients with acne vulgaris and 40 subjects without acne to determine the association between acne severity and BMI [19]. In another study conducted by Tsai et al. on 3274 children, BMI values were significantly higher in patients with acne than that in non-acne group which have suggested that BMI is a risk factor for acne [43]. However, Lu et al. showed that BMI is negatively associated with the number of acne lesions in Taiwanese women with moderate to severe post-adolescent acne in those without PCOS probably advocating BMI and acne relationship varies by age [44]. Although several studies have revealed a link between BMI and acne, no association between those could be found in this study. These results could be considered as a contradictory to those previously published in the literature and this contradiction should be discussed in line with the methodology and target group. Whether serum lipids are elevated in patients with acne or not is also a matter of debate and controversy. In 2010, Arora et al. revealed that compared with controls, adult women with acne had higher levels of serum TC and LDL, lower levels of HDL [40]. In 2007, El-Akawi et al. indicated that patients with acne had significantly 
low plasma HDL levels while TC and TG levels did not differ significantly [45]. As for our results, we have failed to show any significant differences of serum TC, TG, HDL, LDL levels between patients and controls.

Prolactin is a hormone mainly involved in milk production. In contrast to the effects of PRO, PRL increases the 5-ARD enzyme. Darley et al. found hyperprolactinemia in $45 \%$ of 38 women patients with late onset or persistent acne vulgaris [46]. 20 women with idiopathic hyperprolactinemia and acne were treated with bromocriptine by Peserico et al. in 1988 . Both serum PRL levels and acne lesions showed a great improvement with this drug in this study [47]. In terms of serum PRL, we found no significant differences between female groups. This is a field that needs further investigation on larger number of cases with analyses performed on different days. The role of FSH and LH in acne vulgaris is also not well-documented. In a study from Italy, 6 women with acne and 6 women with hirsutism were treated by Faloia et al. with a GnRH analog which suppresses FSH and LH levels. During the treatment, clinical score for acne showed a significant reduction revealing the hypothesis that suppressing ovarian steroids by GnRH analogs might be an effective treatment in women suffering from acne [48]. Combined oral contraceptives which are particularly used for adult female acne treatment, inhibit FSH and LH release in the pituitary via negative feedback loops and thereby androgen production suppression and acne improvement [32]. Besides, PCOS patients may show increased $\mathrm{LH} / \mathrm{FSH}$ ratio in which acne is a common clinical finding in these patients [20]. A study from Turkey on 60 patients with acne and 30 controls showed that serum FSH and LH levels measured on $5^{\text {th }}$ day of menstruative cycle did not differ between groups [49]. In our study, no patient was diagnosed with PCOS and serum LH and FSH levels did not differ significantly between female cases and controls.

Limiting aspects of this study are its retrospective design, reliance on patient data registered on hospital system, restriction to a single center and being done on a young cohort. Effect of other androgens such as dehydroepiandrosterone (DHEA), dehydroepiandrosterone sulfate (DHEA-S), androstenedione, DHT on acne and assessments performed on different days of menstruative cycle needs further evaluation. Despite the findings demonstrating a relation between serum EST levels and acne, the study limitations do not allow straightforward comment that estradiol is good for acne.

\section{CONCLUSION}

In conclusion, in this study we showed that the female acne patients may have low serum EST levels compared with those of female controls. Further investigation on this topic is required to define the association between serum EST levels and acne. This may also bring a new approach to the treatment of female acne vulgaris by estrogen containing hormone therapies. Further studies are needed to clarify the role of conditions which can decrease and increase EST levels on acne improvement and deterioriation.

\section{Statement of Human and Animal Rights}

All procedures followed were in accordance with the ethical standards of the responsible committee on human experimentation (institutional and national) and with the Helsinki Declaration of 1975, as revised in 2008.

\section{Statement of Informed Consent}

Informed consent was obtained from all patients for being included in the study.

\section{REFERENCES}

1. Zaenglein LA, Thiboutot MD. Acne Vulgaris. In: Bolognia JL, Jorizzo JL, Schaffer JV, editors. Dermatology. $3^{\text {rd }}$ edition. London: Saunders, 2012:545-9.

2. Das S, Reynolds RV. Recent advances in acne pathogenesis: implications for therapy. Am J Clin Dermatol. 2014;15:479-88.

3. Harper JC. An update on the pathogenesis and management of acne vulgaris. J Am Acad Dermatol. 2004;51(1 Suppl):S36-8.

4. Melnik BC. Linking diet to acne metabolomics, inflammation, and comedogenesis: an update. Clin Cosmet Investig Dermatol. 2015;8:371-88.

5. Bowe WP, Joshi SS, Shalita AR. Diet and acne. J Am Acad Dermatol. 2010;63:124-41.

6. Wolkenstein P, Misery L, Amici JM, Maghia R, Branchoux S, Cazeau C, et al. Smoking and dietary factors associated with moderateto-severe acne in French adolescents and young adults: results of a survey using a representative sample. Dermatology. 2015;230:34-9.

7. Abulnaja KO. Changes in the hormone and lipid profile of obese adolescent Saudi females with acne vulgaris. Braz J Med Biol Res. 2009;42:501-5.

8. Ghosh S, Chaudhuri S, Jain VK, Aggarwal K. Profiling and hormonal therapy for acne in women. Indian J Dermatol. 2014;59:107-15.

9. Arora MK, Yadav A, Saini V. Role of hormones in acne vulgaris. Clin Biochem. 2011;44:1035-40.

10. Emiroğlu N, Cengiz FP, Kemeriz F. Insulin resistance in severe acne vulgaris. Postepy Dermatol Alergol. 2015;32:281-5.

11. Cetinözman F, Aksoy DY, Elçin G, Yıldız BO. Insulin sensitivity, androgens and isotretinoin therapy in women with severe acne. J Dermatolog Treat. 2014;25:119-22.

12. Melnik BC, John SM, Plewig G. Acne: risk indicator for increased body mass index and insulin resistance. Acta Derm Venereol. 2013;93:644-9. 
13. Cho EB, Ha JM, Park EJ, Kim KH, Kim KJ. Heredity of acne in Korean patients. J Dermatol. 2014;41:915-7.

14. Proud CG. mTOR Signalling in Health and Disease. Biochem Soc Trans. 2011;39:431-6.

15. Melnik BC. Excessive Leucine-mTORC1-Signalling of Cow MilkBased Infant Formula: The Missing Link to Understand Early Childhood Obesity. J Obes. 2012;2012: 197653.

16. Vowels BR, Yang S, Leyden JJ. Induction of proinflammatory cytokines by a soluble factor of Propionibacterium acnes: implications for chronic inflammatory acne. Infect Immun. 1995;63:3158-65.

17. Castell JV, Gómez-Lechón MJ, David M, Fabra R, Trullenque R, Heinrich PC. Acute-phase response of human hepatocytes: regulation of acute-phase protein synthesis by interleukin- 6 . Hepatology. 1990;12:1179-86.

18. Felker GM, Allen LA, Pocock SJ, Shaw LK, McMurray JJ, Pfeffer MA, et al. Red cell distribution width as a novel prognostic marker in heart failure: data from the CHARM Program and the Duke Databank. J Am Coll Cardiol. 2007;50:40-7.

19. Seleit I, Bakry OA, Abdou AG, Hashim A. Body mass index, selected dietary factors, and acne severity: are they related to in situ expression of insulin-like growth factor-1? Anal Quant Cytopathol Histpathol. 2014;36:267-78.

20. Moura HH, Costa DL, Bagatin E, Sodré CT, Manela-Azulay M. Polycystic ovary syndrome: a dermatologic approach. An Bras Dermatol. 2011;86:111-9.

21. Esmaeilzadeh S, Andarieh MG, Ghadimi R, Delavar MA. Body mass index and gonadotropin hormones ( $\mathrm{LH} \& \mathrm{FSH}$ ) associate with clinical symptoms among women with polycystic ovary syndrome. Glob J Health Sci. 2014;7:101-6.

22. Cassidy DM, Lee CM, Laker MF, Kealey T. Lipogenesis in isolated human sebaceous glands. FEBS Lett. 1986;200:173-6.

23. Katsuta Y, Iida T, Inomata S, Denda M. Unsaturated fatty acids induce calcium influx into keratinocytes and cause abnormal differentiation of epidermis. J Invest Dermatol. 2005;124:1008-13.

24. Strauss JS, Krowchuk DP, Leyden JJ, Lucky AW, Shalita AR, Siegfried EC, et al. Guidelines of care for acne vulgaris management. J Am Acad Dermatol. 2007;56:651-63.

25. Strauss JS, Kligman AM, Pochi PE. The effect of androgens and estrogens on human sebaceous glands. J Invest Dermatol. 1962;39:139-55.

26. Sweeney TM, Szarnicki RJ, Strauss JS, Pochi PE. The effect of estrogen and androgen on the sebaceous gland turnover time. J Invest Dermatol. 1969;53:8-10.

27. Eknoyan G. Adolphe Quetelet (1796-1874)--the average man and indices of obesity. Nephrol Dial Transplant. 2008;23:47-51.

28. BMI classification. World Health Organization. Available at http:// apps.who.int $/ \mathrm{bmi} /$ index.jsp?introPage $=$ intro_3.html. Accessed on December 7, 2017.

29. Van Hooff MH, Voorhorst FJ, Kaptein MB, Hirasing RA, Koppenaal C, Schoemaker J. Predictive value of menstrual cycle pattern, body mass index, hormone levels and polycystic ovaries at age 15 years for oligo-amenorrhoea at age 18 years. Hum Reprod. 2004;19: 383-92.

30. De Benoist B, McLean E, Egli I, Cogswell M, editors. Worldwide prevalence of anaemia 1993-2005. WHO Global Database on Anaemia. Geneva, Switzerland. Available at http://whqlibdoc.who .int/publications/2008/9789241596657_eng.pdf. Accessed on December 7, 2017.

31. Graber Emmy, author. Dellavalle RP, Dahl MV, editors. Hormonal therapy for women with acne vulgaris. Available at http://www. uptodate.com/contents/hormonal-therapy-for-women-with-acne-
vulgaris\#H797718678. Accessed on December 7, 2017.

32. George R, Clarke S, Thiboutot D. Hormonal therapy for acne. Semin Cutan Med Surg. 2008;27:188-96.

33. Huber J, Walch K. Treating acne with oral contraceptives: use of lower doses. Contraception. 2006;73:23-9.

34. Wei B, Qu L, Zhu H, Xiao T, Wei HC, Chen HD, et al. Higher $17 \alpha$-hydroxyprogesterone levels aggravated the severity of male adolescent acne in Northeast China. Dermatology. 2014;229:359-62.

35. Schmidt JB, Spona J, Huber J. Androgen receptor in hirsutism and acne. Gynecol Obstet Invest. 1986;22:206-11.

36. Rabe T, Kowald A, Ortmann J, Rehberger-Schneider S. Inhibition of skin 5 alpha-reductase by oral contraceptive progestins in vitro. Gynecol Endocrinol. 2000;14:223-30.

37. Bitzer J, Tschudin S, Alder J; Swiss Implanon Study Group. Acceptability and side-effects of Implanon in Switzerland: a retrospective study by the Implanon Swiss Study Group. Eur J Contracept Reprod Health Care. 2004;9:278-84.

38. Pochi PE. Acne in premature ovarian failure. Reestablishment of cyclic flare-ups with medroxyprogesterone acetate therapy. Arch Dermatol. 1974;109:556-7.

39. Bakry OA, El Shazly RM, El Farargy SM, Kotb D. Role of hormones and blood lipids in the pathogenesis of acne vulgaris in non-obese, non-hirsute females. Indian Dermatol Online J. 2014;5(Suppl 1):S9-S16.

40. Arora MK, Seth S, Dayal S. The relationship of lipid profile and menstrual cycle with acne vulgaris. Clin Biochem. 2010;43:1415-20.

41. Kaymak Y, Adisen E, Ilter N, Bideci A, Gurler D, Celik B. Dietary glycemic index and glucose, insulin, insulin-like growth factor-I, insulin-like growth factor binding protein 3, and leptin levels in patients with acne. J Am Acad Dermatol. 2007;57:819-23.

42. Kartal D, Yildiz H, Ertas R, Borlu M, Utas S. Association between isolated female acne and insulin resistance: a prospective study. G Ital Dermatol Venereol. 2016;151:353-7.

43. Tsai MC, Chen W, Cheng YW, Wang CY, Chen GY, Hsu TJ. Higher body mass index is a significant risk factor for acne formation in schoolchildren. Eur J Dermatol. 2006;16:251-3.

44. Lu PH, Hsu CH. Body mass index is negatively associated with acne lesion counts in Taiwanese women with post-adolescent acne. J Eur Acad Dermatol Venereol. 2015;29:2046-50.

45. El-Akawi Z, Abdel-Latif N, Abdul-Razzak K, Al-Aboosi M. The Relationship between Blood Lipids Profile and Acne. J Health Scien. 2007;53:596-9.

46. Darley CR, Kirby JD, Besser GM, Munro DD, Edwards CR, Rees LH. Circulating testosterone, sex hormone binding globulin and prolactin in women with late onset or persistent acne vulgaris. Br J Dermatol. 1982;106:517-22.

47. Peserico A, Ruzza G, Veller Fornasa C, Bertoli P, Cipriani R. Bromocriptine treatment in patients with late onset acne and idiopathic hyperprolactinemia. Acta Derm Venereol. 1988;68:83-4.

48. Faloia E, Filipponi S, Mancini V, Morosini P, De Pirro R. Treatment with a gonadotropin-releasing hormone agonist in acne or idiopathic hirsutism. J Endocrinol Invest. 1993;16:675-7.

49. Aksoylar NA, Arıcan Ö, Koldaş M, Karaoğlu A. Serum sex hormone levels in female patients with acne vulgaris. Kartal Ed Train Hosp J Med. 2002;13:9-13.

Copyright by Neslihan Akdoğan, et al. This is an open-access article distributed under the terms of the Creative Commons Attribution License, which permits unrestricted use, distribution, and reproduction in any medium, provided the original author and source are credited.

Source of Support: Nil, Conflict of Interest: None declared. 